\title{
Docencia de pregrado en medicina interna en un hospital privado: Diez años de experiencia
}

\author{
Antonio Vukusich C ${ }^{1}$, Ricardo Larrea $\mathbf{G}^{1}$, Mauricio Álamo $\mathrm{T}^{1}$, \\ Juan Carlos Ponce $\mathbf{M}^{1}$, G onzalo Valls $\mathbf{G}^{1}$, \\ Claudio G onzález $\mathrm{T}^{1}$, Francisco Bidegain $\mathrm{G}^{1}$, \\ Marcos $G$ utiérrez $M^{1}$, Mario Rivera $K^{1}$, Ángel Vargas $D^{1}$, \\ Carlos $N$ avarrete $M^{1}$, Jonás $\mathbf{G}$ óngora $R^{1}$, Luis $\mathbf{G}$ oldin $\mathbf{G}^{1}$, \\ Cristian Varela U ${ }^{1}$, Pamela Bozzo ${ }^{2}$, Rogelio Altuzarra $H^{2}$. \\ Undergraduate teaching of internal
medicine in a private hospital
}

There is a 10 years teaching experience for fourth year medical students and interns in a Chilean private hospital. The students attend an eight weeks practical course. The interns rotate during 16 weeks by specialties and make shifts. The hospital structure with Clinical Services and Medical-Surgical departments facilitates the teaching process. There are approximately 30,000 admissions per year with a mean stay of 3.7 days, that allow the students to be in touch with patients with different diseases that are managed with updated technology. We emphasize the ethical and clinical management of concrete problems of patients, self learning and communication skills. The students evaluate their stay answering surveys and with semi structured interviews. Teaching is assessed by tutors and heads of departments, in clinical rounds, sometimes prepared by the students, by a thorough revision of problem oriented medical records and with practical and theoretical tests. The results of the program have been quite satisfactory for participants (Rev Méd Chile 2009; 137: 1105-12).

(Key words: Education, medical, undergraduate; Hospitals, private; Internal Medicine)

Recibido el 6 de agosto, 2008. Aceptado el 6 de enero, 2009.

${ }^{1}$ Clínica Dávila y Departamento de Medicina Interna, Facultad de Medicina Universidad de los Andes. Santiago de Chile. ${ }^{2}$ Facultad de Medicina Universidad de los Andes

$\mathrm{P}$ or diversas razones, durante los últimos años, el número de Escuelas de Medicina se ha multiplicado y los campos clínicos idóneos para una educación médica completa se han vuelto escasos $^{1,2}$. La situación es nueva y demanda un abordaje creativo. Chile tiene un sistema de salud mixto, mayoritariamente público, que tradicional-

Correspondencia a: Dr. Antonio Vukusich Covacic. Clínica Dávila. Recoleta 464, Santiago. Teléfono: 7308021. Fax: 7308885. E mail: dirmedica@davila.cl mente ha sostenido la docencia, pero cuenta, también, con un sector privado consolidado que hasta ahora ha participado muy poco en ella ${ }^{3}$.

Este artículo comunica una experiencia de 10 años de docencia de pregrado en medicina interna en Clínica Dávila, uno de los campos clínicos para estudiantes de $4^{\circ}$ año e internado de la Universidad de los Andes. Primero describiremos los aspectos estructurales y organizativos del hospital que posibilitan la docencia, luego, los objetivos comunes de ambos programas y, finalmente, los objetivos pedagógicos, metodología y resultados de cada uno de ellos. 


\section{CARACTERÍSTICAS DEL HOSPITAL QUE FACILITAN LA DOCENCIA}

Un hospital adquiere el carácter de "docente" cuando consigue mantener, de forma prolongada, un ambiente apropiado para el proceso de enseñanza-aprendizaje. Con este propósito in mente decidimos partir por explicitar la vocación docente de nuestro hospital tanto en la descripción de su misión como en el Estatuto del Cuerpo Médico que describe la organización, privilegios y obligaciones de los profesionales relacionados con la institución. Junto con esto, pusimos especial cuidado en que la estructura, organización, modelo de gestión y características de los docentes, fueran adecuadas y ejemplares para la formación de los alumnos.

\section{Estructura organizacional y magnitud de la} actividad. Clínica Dávila es un hospital complejo con un alto nivel de informatización de los procesos, incluyendo el registro clínico electrónico de la mayor parte de la actividad. En los últimos años ha tenido un crecimiento sostenido pasando de 160 a 404 camas con una ocupación mayor a 80\%. La estada promedio global es de 3,7 días. Esto resulta en cerca de 30.000 egresos anuales lo que sitúa a la Clínica entre los 10 hospitales con mayor actividad del país. La capacidad resolutiva es prácticamente total, incluyendo cirugía cardiaca y trasplantes. Para la docencia en medicina interna disponemos de 18 camas de Cuidados Intensivos, 30 de Tratamiento Intermedio, 14 de Coronaria y 170 de MédicoQuirúrgico. El Servicio de Urgencia atiende más de 40.000 pacientes adultos/año y las consultas electivas ambulatorias superan las 200.000.

Para la gestión clínica y docencia el hospital está estructurado en unidades territoriales y funcionales. Las primeras corresponden a Servicios que disponen de un espacio físico (Urgencia, Cuidados Intensivos, Radiología, etc.) En el diseño de cada Servicio se ha considerado, cuidadosamente, que la infraestructura sea adecuada para el trabajo de internos y alumnos, incluyendo salas de reuniones y descanso, residencias, biblioteca, auditorios, conexiones Internet.

Las unidades funcionales están constituidas como Departamentos médico-quirúrgicos que, reconociendo la interdependencia de ambos aspectos de la práctica clínica, formalizan su integración estimulando el trabajo en equipo. Los Departamentos organizan y regulan todas las actividades relacionadas con una $\mathrm{O}$ más especialidades cercanas entre sí y materializan sus funciones en las unidades territoriales. Tanto los Servicios, como los Departamentos, son dirigidos por jefaturas médicas responsables de promover y practicar la docencia e investigación clínica.

2. Administración médica y docencia. La Dirección Médica ha definido cuatro áreas estratégicas: Acreditación institucional; Planificación y Proyectos clínicos; Gestión de riesgos; Docencia y Desarrollo. Desde sus perspectivas particulares los Directores de Áreas conducen de forma transversal el quehacer de los Servicios y Departamentos y se integran en un Comité Clínico Ejecutivo donde, junto con los demás aspectos de la gestión, el estado de los programas docentes es monitorizado y evaluado.

\section{Características del cuerpo médico y modelo de} atención. Actualmente contamos con 400 médicos staff de los cuales 102 son internistas/subespecialistas responsables de más de 95\% de la atención. El resto depende de médicos externos acreditados y regidos por el Estatuto del Cuerpo Médico. Cada médico pertenece a un Departamento, reconoce una jefatura y es evaluado por el área de Docencia y Desarrollo la que asigna un puntaje mayor a quienes realizan labores docentes o de investigación. El 50\% de los médicos staff tiene experiencia docente y posee un grado académico.

El modelo de atención considera una relación médico-paciente-familia personalizada. Todo paciente, independiente del lugar de hospitalización, seguro de salud, o cualquier otra condición, tiene un médico tratante responsable, las $24 \mathrm{~h}$ del día, de conducir el caso y de comunicarse con la familia. Los honorarios, que se acuerdan entre los médicos y el hospital, se cobran al seguro de salud del paciente. Los residentes y las jefaturas médicas tienen el carácter de funcionarios remunerados por la Clínica, pero suelen tener, además, la condición de médicos tratantes.

4. Medición de resultados y control de calidad. Los resultados de la actividad clínica se gestionan en base a un centenar de indicadores objetivos. Para estandarizar la atención y disminuir la variabilidad no deseada de la práctica hemos editado 120 Guías Clínicas que están al alcance de los médicos tratantes, docentes y alumnos. Disponemos de un registro anónimo computarizado de incidentes de acceso inmediato a todo el personal, auditoría médica y comités de Revisión de Prácticas Clínicas y de Mejoría Continua. 
5. Convenio con la Universidad. Las relaciones entre la clínica y la universidad se formalizan a través de un convenio que regula el quehacer de los estudiantes durante su permanencia en el hospital. La Universidad es la encargada de remunerar a los médicos staff que son académicos, se hace cargo de los gastos de alimentación y uso de las instalaciones y concurre con fondos para el desarrollo de la infraestructura necesaria para la docencia.

6. Características de los docentes. La actividad docente es optativa y depende de los intereses, habilidades y competencias desarrolladas. Participan en ella los médicos tratantes que lo solicitan, y se mantienen ejerciéndola quienes son bien evaluados. Privilegiamos en nuestros docentes un espíritu constructivo y esperanzador, la dedicación y compromiso con los alumnos, la integridad personal y profesional, el sentido del humor y, sobre todo, el mayor interés por el cuidado solícito de los pacientes. Gran parte de los docentes han asistido a congresos, cursos, seminarios o diplomados de docencia en medicina. Realizamos reuniones periódicas para evaluar cada programa y compartir inquietudes o soluciones creativas, teniendo a la vista los resultados que cada grupo de alumnos va obteniendo y que determinan los cambios que parecen apropiados para las siguientes rotaciones.

7. Información a los pacientes. Los pacientes, o sus representantes, son informados sobre la condición docente del hospital por medio del Reglamento de Hospitalización, la Carta de Derechos del Paciente, los médicos tratantes y docentes, la credencial que portan los alumnos identificando su condición y por folletos institucionales. Al tomar contacto con ellos o sus tutores, los pacientes deben dar su consentimiento verbal explícito para seguir adelante con la entrevista, el examen o la visita; de otra manera, el proceso debe suspenderse o seguir caminos alternativos (análisis de la ficha, patología, laboratorio o imágenes) a la espera de una mejor ocasión. Procuramos ser extraordinariamente prudentes en la oportunidad, número y duración de las visitas clínicas de más de dos o tres personas.

\section{Características COMUNES DE AMBOS PROGRAMAS}

Teniendo a la vista las competencias requeridas para un médico del siglo XXI, en ambos programas se ha puesto énfasis en los siguientes aspectos: 1. El desarrollo del profesionalismo y las habilidades comunicacionales, como el mejor camino para lograr pacientes y médicos satisfechos ${ }^{4-8}$. Reforzamos los talleres de comunicación y técnica de entrevista que se inician en $3^{\circ}$ año como parte de una línea de investigación para que, como se hace con el diagnóstico y tratamiento, la entrega de información a los pacientes sea planificada? ${ }^{9}$. El autoaprendizaje. Consideramos al alumno el motor de la formación y estimulamos una actitud proactiva y el buen uso de los medios docentes disponibles. 3. El manejo ético de los pacientes a partir de sus problemas clínicos concretos y no sólo de diagnósticos hipotéticos que suelen terminar en generalizaciones teórico-académicas $^{10,11}$. 4. La capacidad de buscar evidencia en forma rápida para la toma de decisiones.

Cada programa tiene un coordinador responsable de interactuar con las jefaturas médicas, docentes, Dirección de Docencia y autoridades de la Facultad.

\section{Objetivos y método Curso Práctico de Medicina $4^{\mathrm{O}}$ AÑO}

Los objetivos generales y la metodología se resumen en las Tablas 1 y 2 . Para lograr los objetivos cognoscitivos de este nivel los alumnos dedican 4 horas diarias al conocimiento sistemático de sus pacientes, incluyendo, el adiestramiento para buscar información que permita: analizar los problemas de los enfermos y la racionalidad de las decisiones, la lectura crítica de artículos seleccionados y la preparación, en grupo, de reuniones clínicas de casos frecuentes, interesantes o complejos para practicar el trabajo en equipo. Esperamos que los alumnos aumenten sus habilidades y destrezas en el examen clínico, ejercicio diagnóstico, terapéutica, interpretación de exámenes de laboratorio e imágenes y que logren una relación adecuada con los pacientes y sus familias. La realización de historias semanales, orientadas por problemas, ha sido una herramienta importante para objetivar ciertos logros ${ }^{12-14}$.

Los objetivos actitudinales enfatizan la preocupación efectiva por la intimidad de los enfermos, su autonomía y el secreto profesional. Nos parece esencial el uso adecuado del lenguaje médico-social, evitando prejuzgar o criticar con liviandad las decisiones de otros profesionales y evitar conductas 


\section{Tabla 1. 0 bjetivos generales curso práctico medicina interna $4^{\circ}$ año}

1. Obtener historias y exámenes físicos confiables.

2. Conocer etiología, patogenia, anatomía patológica, fisiopatología, clínica, diagnóstico y tratamiento de patologías vistas.

3. Interpretar exámenes de laboratorio e imágenes y conocer su utilidad y limitaciones.

4. Plantear diagnósticos sindromáticos, fisiopatológicos, patogénicos, etiológicos y diferenciales.

5. Proponer planes diagnósticos y terapéuticos a partir de los problemas de los pacientes.

6. Planificar contenido y oportunidad de información a entregar a pacientes y familiares.

\section{Tabla 2. Aspectos metodológicos curso práctico medicina interna $4^{\circ}$ año}

1. Relación docente/alumno: $1 / 5$.

2. $\mathrm{N}^{\circ}$ docentes en el programa: 6 .

3. $\mathrm{N}^{\circ}$ alumnos por pasada: 15 .

4. Servicios o Departamentos utilizados: Todos los de adultos.

5. Duración rotaciones: 8 semanas.

6. Realización de práctica supervisada, estudio diario y exhaustivo de pacientes.

7. Visitas clínicas con docentes.

8. Entrega de historia semanal orientada por problemas.

9. Reuniones clínicas preparadas por alumnos.

10. Seminarios semanales de análisis crítico de la literatura, temas, casos.

arbitrarias o abusivas de los docentes ${ }^{15}$. La presentación y conducta personales deben cumplir con las expectativas de los pacientes y equipo de salud, incluyendo, identificación, puntualidad, desarrollo de hábitos de trabajo personal y grupal, respeto de los reglamentos y cuidado de los bienes del hospital.

EVALUACIÓN $4^{\circ}$ AÑO

Hacemos una evaluación personal, según pauta, de los conocimientos, actitudes, interés, habilidades, participación y calidad de las presentaciones e historias clínicas (70\%) y un examen práctico final (30\%).

\section{OBJETIVOS Y MÉTODO INTERNADO}

Los objetivos se resumen en la Tabla 3. Damos especial importancia a la epidemiología y control de enfermedades prevalentes, como diabetes mellitus e hipertensión arterial y estimulamos la búsqueda de nuevas formas de aproximación preventiva. El hecho de que se trate de un hospital privado, donde los pacientes cancelan un co-pago por su atención, obliga a justificar mejor el uso de exámenes de laboratorio, imágenes, etc., conociendo su sensibilidad, especificidad y las alternativas costo-eficaces. No se realizan estudios diagnósticos ni procedimientos "con fines docentes" y evitamos involucrar a nuestros estudiantes en actividades que carezcan de objetivos pedagógicos precisos ${ }^{10}$. La Tabla 4 resume los aspectos metodológicos.

\section{EvALUACIÓN INTERNADO}

Aplicamos una evaluación personal, según pauta, que considera aspectos actitudinales, cognoscitivos, habilidades y destrezas. Con respecto a los primeros, la responsabilidad, autocrítica y reconocimiento de las debilidades son considerados especialmente. Los internos rinden diversos exámenes teórico-prácticos y un examen final oral de pregrado.

\section{Resultados}

La falta de evaluaciones estructuradas, objetivas y compartidas de la enseñanza clínica de la medicina 
Tabla 3. 0 bjetivos internado medicina interna

1. Reconocer situaciones clínicas complejas y participar de su manejo con equipo tratante.

2. Conocer los modos de prevención de las diferentes enfermedades de medicina interna.

3. Utilizar e interpretar adecuadamente los exámenes de laboratorio e imágenes.

4. Utilizar críticamente la literatura actualizada para la toma de decisiones.

5. Participar en el proceso de planificación y acompañar en la entrega de información al paciente y familiares.

6. Colaborar en la realización de procedimientos invasivos.

7. Lograr los objetivos propios de cada pasada por especialidades.

\section{Tabla 4. M etodología internado medicina interna}

1. $\mathrm{N}^{\circ}$ docentes: 14 .

2. $\mathrm{N}^{\circ}$ internos por pasada: $15-25$.

3. Duración de estadía: 16 semanas.

4. Rotaciones: 2 semanas en: UCI, UTI, Unidad Coronaria, Nefrología, Gastroenterología, Urgencia, Neurología, Médico-Quirúrgico.

5. $\mathrm{N}^{\circ}$ internos por rotación: $2-3$.

6. Reuniones clínicas, bibliográficas, visitas, turnos.

7. Seminarios de especialidades y radiología.

UCI: Unidad de Cuidados Intensivos. UTI: Unidad de Tratamiento Intermedio.

interna, no nos permite comparar con otras experiencias nacionales. Sin embargo, podemos afirmar que, dado el volumen de actividad, los alumnos ven una gran variedad de patologías. Observamos como van adecuando su lenguaje y ampliando sus destrezas clínicas para enfrentar una relación médicopaciente-familia cada día más "horizontal" y exigente. Han logrado incorporarse, de lleno, al proceso de toma de decisiones y manejo de pacientes. La adaptación a la rutina y reglas del hospital ha resultado muy satisfactoria sin alterar el quehacer asistencial. La preparación de reuniones clínicas para médicos tratantes, tutores y, sobre todo pares, ha aumentado sus destrezas para buscar información y el uso crítico de la literatura. Alumnos destacados han presentado trabajos de investigación, realizados en la Clínica, en congresos de estudiantes o de medicina interna. Otros, aparecen junto a sus docentes en publicaciones nacionales.

Las evaluaciones periódicas de ambos programas consideran las siguientes perspectivas:

1. Los pacientes, en entrevistas con los coordinadores de programas, manifiestan, como ha sido reportado, su aceptación y buena disposición hacia los alumnos siempre que no sean ellos los que estén a cargo de la toma de decisiones ${ }^{16,17}$.

2. Los alumnos evalúan el proceso en reuniones individuales y grupales con los coordinadores de programas y por medio de encuestas anónimas que precisan el valor de las diferentes actividades y permiten corregir a tiempo los problemas (Tabla 5).

3. Los docentes han evaluado bien ambos programas, tanto con las autoridades de la Facultad como en reuniones al término de cada pasada donde se analiza, entre otros aspectos, los resultados de la evaluación de los alumnos para comparar entre pares y observar tendencias.

4. Mediciones independientes pueden ser considerados los resultados de los exámenes de pre-grado tomados por la Pontificia Universidad Católica, universidad examinadora, durante el período pre-acreditación (Tabla 6) y el Examen Médico Nacional (Tabla 7).

5. La administración y el personal muestran satisfacción con el programa. El nivel de los costos no ha variado, se valora el "control de calidad" que, tácitamente, ejercen los alumnos y, el 
Tabla 5. Evaluación internado medicina interna (\#)

\begin{tabular}{|lccc|}
\hline Años & $\mathbf{2 0 0 6}$ & $\mathbf{2 0 0 7}$ & $\mathbf{2 0 0 8}$ \\
\hline Pasadas por especialidades (*) & & & \\
$\quad$ Variedad de patologías & 6,0 & 6,1 & 6,0 \\
Calidad/actualidad de tecnología & 6,5 & 6,5 & 6,5 \\
Ocupación del tiempo & 5,8 & 6,0 & 5,9 \\
Calidad docencia & 6,1 & 6,0 & 6,1 \\
Turnos de residencia & 6,0 & 5,9 & 6,0 \\
Ocupación del tiempo & 6,0 & 6,1 & 6,0 \\
Calidad docencia & & 6,0 & 6,6 \\
Ambiente docente & 6,6 & 6,5 & 6,8 \\
Calidad de las residencias, auditorios, biblioteca, etc. & 6,9 & 6,7 & 5,8 \\
Relación con médicos & 5,8 & 5,7 & 6,0 \\
Relación con enfermeras & 6,0 & 6,0 & \\
Impresión general internado & & & \\
\hline
\end{tabular}

(\#) Escala de 1,0 a 7,0. Número de internos encuestados: 98.

(*) Servicios: Unidad de Cuidados Intensivos, Unidad de Tratamiento Intermedio, Urgencia, Médicoquirúrgico. Departamentos: Enfermedades cardiovasculares (Unidad Coronaria), Enfermedades Digestivas, Nefro-Urología, Neurología-Neurocirugía.

Tabla 6. Calificaciones internado medicina interna y exámenes de pregrado. Período pre-acreditación (*)

\begin{tabular}{|lccc|}
\hline Año & N & Internado & Pregrado (\#) \\
\hline 1998 & 21 & 6,1 & 6,2 \\
1999 & 25 & 5,9 & 5,8 \\
2000 & 41 & 6,2 & 6,2 \\
2001 & 49 & 6,2 & 6,2 \\
\hline
\end{tabular}

(*) Promedio de notas, escala de 1,0 a 7,0

(*) La universidad examinadora fue la Pontificia Universidad Católica de Chile.

Tabla 7. Resultados examen médico nacional

\begin{tabular}{|c|c|c|c|c|}
\hline \multirow[b]{2}{*}{ Año } & \multicolumn{2}{|c|}{ Media global (\#) } & \multicolumn{2}{|c|}{ Media medicina interna $\left(^{\#}\right)$} \\
\hline & Nacional & U. Andes & Nacional & U. Andes \\
\hline 2003 & 62,17 & 62,54 & 61,46 & 66,71 \\
\hline 2004 & 63,25 & 68,34 & 62,61 & 71,36 \\
\hline 2005 & 63,30 & 70,26 & 64,30 & 73,13 \\
\hline 2006 & 63,61 & 65,85 & 62,09 & 67,65 \\
\hline 2007 & 63,61 & 69,41 & 71,59 & 76,12 \\
\hline
\end{tabular}

(\#) \% de respuestas correctas. 
registro de incidentes en línea, de fácil acceso a todo el personal, no registra eventos vinculados con la docencia.

Finalmente, el éxito de la docencia en medicina interna ha estimulado a otros Servicios y Departamentos a desarrollar sus propios proyectos. Actualmente, tenemos alumnos de medicina en anestesia, pediatría, obstetricia y cirugía y convenios con diferentes casas de estudios superiores en enfermería, tecnología médica, química y farmacia, kinesiología y nutrición.

\section{DisCUSIÓN}

Se ha sostenido, con razón, que el desarrollo del área salud y la educación médica en Chile, durante el siglo XX, se debieron a la colaboración recíproca entre las universidades y los hospitales públicos $^{3}$. La creación, en ellos, de cátedras universitarias descentralizó la enseñanza y fortaleció la asistencia y la docencia bajo el concepto de relación docente-asistencial ${ }^{18}$. Hoy día, sin embargo, un mayor número de estudiantes, está superando las posibilidades de este modelo. En este escenario, hace más de 10 años, nos propusimos el desafío de transformar a Clínica Dávila en un campo clínico universitario iniciando la docencia para alumnos de $4^{\circ}$ año e internado de medicina interna de la Universidad de los Andes.

Para comenzar un programa de este tipo, que por cierto debe ser progresivo, hay que considerar diferentes aspectos. Las habilidades de las personas y los recursos materiales, esenciales para la enseñanza, dependen de las competencias que se desean obtener, sin embargo, en el pre-grado, el alumno es formado, fundamentalmente, en el modelo biológico de enfermedad que privilegia el conocimiento de los mecanismos patogénicos y la elaboración de diagnósticos hipotéticos. Para conseguir los objetivos pedagógicos que se derivan de esta visión, resultan muy importantes la infraestructura, organización, complejidad, volumen de actividad y variedad de problemas de salud que recibe un hospital; pero también, con qué oportunidad, tecnología y eficiencia es capaz de resolverlos.

Los hospitales privados complejos suelen tener instalaciones adaptables para la docencia, cuentan con una adecuada gestión clínica, tecnología actualizada, controles de calidad y están sometidos a una gran exigencia de pacientes que valoran, cada vez más, el principio de autonomía en la toma de decisiones $^{19}$. Lo anterior, siendo necesario, no es suficiente para lograr un ambiente apropiado para la docencia ya que él depende, más bien, de la calidad de los docentes y del modelo de atención. En nuestro caso, fuimos desarrollando, paulatinamente, tanto la infraestructura que permitiera soportar la enseñanza como una forma de organización del trabajo médico que, estimulando una correcta labor asistencial, fuera compatible con una docencia efectiva.

En general, en los hospitales privados, el peso del manejo asistencial recae en la figura del médico tratante quien, a diferencia del médico funcionario, que cumple un horario, tiene una responsabilidad continua sobre sus pacientes y debe estar permanentemente disponible. En nuestro hospital, el médico tratante pertenece, además, a un Servicio o Departamento que está desarrollando un abordaje sistemático de los problemas clínicos y mide los resultados de su práctica. Este modelo de atención asegura la continuidad del cuidado del paciente, estimula la interacción entre miembros de un mismo Departamento o Servicio, o con los de otros, y favorece un ambiente docente. Por otra parte, difiere de la relación docente-asistencial tradicional ya que puede prescindir de los alumnos para la rutina asistencial y éstos pueden dedicarse más al aprendizaje que a la asistencia.

Pensamos que el médico tratante, con vocación docente, es el más indicado para crear una atmósfera adecuada para la transferencia de conocimientos y destrezas. Y no sólo en el ámbito clínico. El médico tratante, que realiza una práctica prudente, mientras apoya y consuela a sus pacientes, encarna un modelo social fundamental en la formación de los alumnos como profesionales y ciudadanos ${ }^{1,8,10}$. Puede parecer sorprendente que este modus operandi sea aplicable, en nuestros días, en un hospital privado pero no es algo nuevo. Ya la escuela hipocrática nos revela al médico rodeado de discípulos, actuando y enseñando como si ambos quehaceres fueran uno ${ }^{20}$.

Los buenos resultados de nuestra experiencia nos han hecho pensar que el adecuado ejercicio de la medicina no puede "liberarse" del proceso de enseñanza-aprendizaje. Por una parte, el médico, obligado a actualizarse, debe ser, literalmente, 
un estudiante toda su vida. Y, por otra, el amplio interés y disponibilidad docente de los médicos parece deberse, al menos en parte, a que perciben en el enseñar la mejor manera de mantener vigentes sus conocimientos y destrezas.

La literatura describe un número y variedad limitados de experiencias docentes de pre-grado exitosas en hospitales privados de Europa, Estados Unidos de Norteamérica, Australia y Asia ${ }^{21-25}$. La aceptación de los estudiantes por parte de los pacientes, que podría ser un problema, no parece tal. Nuestra experiencia es concordante con un estudio nacional que mostró que ellos están dispuestos a colaborar, bajo condiciones de seguridad, universalmente deseables, con la docencia

\section{ReFERENCIAS}

1. Gorc A. Proliferación de escuelas de medicina en Latinoamérica: causas y consecuencias. Rev Méd Chile 2002; 130: 917-24.

2. Свотту B. More students and less patients: the squeeze on medical teaching resources. Med J Aust 2005; 183: 444-5.

3. Goic A, Armas R. Descentralización en salud y educación: La experiencia chilena. Rev Méd Chile 2003; 131: 788-98.

4. Rennie S. The medical students in the Year 2020. Medical Teacher 2000; 22: 532-5.

5. Rosselot E. Planificando la educación médica para las próximas décadas. Rev Méd Chile 2001; 129: 1473-8.

6. Vukusich A, Olivari F, Alvarado N, Burgos G. Causas de la crisis de la profesión médica en Chile. Rev Méd Chile 2004; 132: 1425-30.

7. Rosselot E. Hacia el médico que nuestros países necesitan: énfasis en la comunicación y en la formación de los docentes. Rev Méd Chile 2003; 131: 331-7.

8. Rosselot E. Dimensiones del profesionalismo médico. Proyecciones para el siglo XXI. Rev Méd Chile 2006; 134: 657-64.

9. Florenzano R, Altuzarra R, Carvajal C, Weil K, Dörr A, Fullerton C et al. Mejorando la calidad de la enseñanza de entrevista clínica: evaluación de una intervención en estudiantes de medicina. Rev Méd Chile 2000; 128: 294-300.

10. Spencer J, Blackmore D, Heard S, Mc Crorie P, Mc Haffie D, SCherpbier A ET AL. Patient-oriented learning: a review of the role of the patient in the education of medical students. Med Educ 2000; 34: 851-7.

11. Doyal L. Closing the gap between professional teaching and practice. BMJ 2001; 322: 685-6.

12. WeEd L. Medical Record that Guide and Teach. N Engl J Med 1968; 278: 593-600. en hospitales privados. Estos, por su parte, asumen el deber de aumentar su garantía de calidad y la satisfacción de los usuarios ${ }^{16}$.

Concluimos que la enseñanza clínica de pregrado en medicina interna, tanto a nivel de $4^{\circ}$ año como del internado, es posible y exitosa en un hospital privado estructurado bajo las condiciones mencionadas.

\section{Agradecimientos}

Los autores agradecen a las siguientes personas sus comentarios y sugerencias los que fueron de gran utilidad en la preparación de este trabajo: Srs.: Eduardo Urrutia H. y Mario Rivas S. y Drs.: Paula Daza N., Francisco Olivari P. y Cynthia Vukusich V.

13. Friedman H. Problem-Oriented medical Diagnosis. Boston. Little, Brown and Company. 1981.

14. VuKusich A, VAldés S. La ficha clínica orientada por problemas. Fundamentos de su aplicación en enfermos críticos. Rev Méd Chile 1988; 116: 1070-4.

15. Maida A, Herskovic V, Pereira A, Salinas-Fernández L, Esquivel C. Percepción de conductas abusivas en estudiantes de medicina. Rev Méd Chile 2006; 134: 1516-23.

16. Beca JP, Browne F, Valdebenito C, Bataszew V, Martinez MJ. Relación estudiante-enfermo: Visión del paciente. Rev Méd Chile 2006; 134: 955-9.

17. Spencer J. Patients in medical education. The Lancet 2004; 363: 1480.

18. Goic A. El modelo chileno de relación docenteasistencial. Rev Méd Chile 1975; 233: 466-7.

19. Pellegrino E. La metamorfosis de la ética médica. Una mirada retrospectiva a los últimos 30 años. En: Cuadernos del Programa Regional de Bioética. OPS, OMS. 1995; 35-53.

20. Lain P. Historia de la Medicina. Barcelona: Salvat, 1982; 91-110.

21. Mahal A, Mohanan M. Growth of private medical education in India. Med Educ 2006; 40: 1009-11.

22. BeIsCher N. Private patients and undergraduate teaching of obstetrics in Victoria. Med J Aust 1973; 1: 507-9.

23. Parkerson G, Michener J, Muhlbaier L, Falcone J. Clinical experience of medical students in model family practices and private family practices. J Fam Pract 1986; 23: 361-6.

24. Tschudi P, Bally K, Isler R. One-on-one tutorials in private practices and clinics. Four years of experience in Basel, Switzerland. Med Teach 2003; 25: 537-43.

25. Mc Curdy F, Sell D, Beck G, Kerber K, Larzelere R, Evans J. A comparison of clinical pediatric patient encounters in university medical center and community private practice settings. Am Pediatr 2003; 3: 12-5. 